\title{
Qualidade de Vida de Adolescentes Modelos profissionais
}

\author{
Luciana Pires \\ Centro de Estudos e Pesquisas Sanny \\ Alexandra Magna Rodrigues \\ Universidade de Taubaté \\ Mauro Fisberg \\ Universidade Federal de São Paulo \\ Roberto Fernandes da Costa \\ Centro de Estudos e Pesquisas Sanny \\ Teresa Helena Schoen ${ }^{1}$ \\ Universidade Federal de São Paulo
}

\begin{abstract}
RESUMO - Muitas adolescentes deixam suas famílias para seguir a profissão de modelo, sonhando com um futuro glamoroso. O presente estudo caso-controle analisou a qualidade de vida de 74 adolescentes do sexo feminino, sendo 37 modelos, agenciadas em São Paulo, com delineamento transversal, utilizando o World Health Organization Quality of Life - versão breve (WHOQOLBREF), que avalia qualidade de vida global e os domínios físico, psicológico, social e ambiental. Utilizou-se o Critério Brasil 2008, para avaliação do nível socioeconômico e para parear o grupo controle. Em geral, o grupo de modelos obteve médias superiores ao grupo de não modelos, sendo esta diferença significante apenas no domínio psicológico. Observou-se que as adolescentes modelos apresentaram uma qualidade de vida semelhante à das não modelos.
\end{abstract}

Palavras-chave: Adolescente; qualidade de vida, Comportamento do adolescente, saúde mental do adolescente, trabalho juvenil

\section{Quality of Life of Professional Adolescent Models}

\begin{abstract}
Many adolescent girls leave their families to pursue a modeling career, dreaming of a glamorous future. The present cross-sectional case-control study analyzed the quality of life of 74 female adolescents, 37 of whom were models at agencies in the city of São Paulo (Brazil), using the World Health Organization Quality of Life -BREF questionnaire, which analyzes physical, psychological, social and environmental domains. The Brazil Criteria 2008 were used for the assessment of socioeconomic status and for pairing the control group. The group of models generally achieved higher mean scores than the non-model group, with the difference only significant on the psychological subscale. The results show that the teenage models had a similar quality of life to that of the non-models.
\end{abstract}

Keywords: Adolescents; quality of life; teenage behavior; mental health of adolescents; youth employment

Embora a adolescência seja um fenômeno universal, possui características bastante particulares, que variam de indivíduo para indivíduo, sofrendo influência do ambiente social, cultural e econômico (Schoen-Ferreira, Aznar-Farias \& Silvares, 2010). Características como a crescente independência, alterações psicológicas, busca de autonomia, definição da própria identidade, influência de amigos, demandas escolares e de trabalho, pressões e modificação das preferências alimentares, fazem deste um grupo que pode apresentar possíveis riscos em todos os setores da sua atividade (Liborio \& Ungar, 2010; Mota \& Matos, 2009; Pfeifer, Martins \& Santos, 2010; Schoen-Ferreira, Aznar-Farias \& Silvares, 2009). Embora o adolescente esteja mais independente, a família ainda tem o dever de cuidar e proteger este membro em desenvolvimento. Esta proteção aos poucos vai sendo retirada, entretanto ainda aparece em diversas situações, como ao acompanhar o adolescente ao médico e matriculá-lo na escola (Schoen-Ferreira \& Vitalle, no prelo).

1 Endereço para correspondência: Rua Joaquim Távora, 550 apto $84 \mathrm{~B}$ CEP: 04015-011 São Paulo-SP; E-mail: rpetrass@uol.com.br
É nesta época que meninos e meninas têm a liberdade e a responsabilidade da escolha. Alguns escolhem seguir a profissão dos pais, outros serem atletas, bailarinos, modelos ou outra profissão (Noronha \& Ambiel, 2006; Rodrigues, 2003). Muitas adolescentes sonham com um futuro promissor e encantador que a profissão modelo oferece. Este sonho faz parte, principalmente, do universo feminino (Rodrigues, 2003). Modelo profissional, no sentido mais restrito, significa modelo de beleza. No sentido mais amplo, modelo profissional seria toda pessoa que serve para representar um produto, seja na moda ou publicidade. No mundo da moda se exige dos modelos corpos esguios e bonitos, rostos interessantes, cabelos e peles bem cuidados. Já na publicidade, as exigências são outras e dependem do tipo de produto que se quer vender (Libardi, 2004). O olhar fica restrito à aparência física do indivíduo, sem preocupação com outras áreas do desenvolvimento (Calatayud Arenes \& Serra Desfilis, 2009; Sarmento, Schoen-Ferreira, Medeiros \& Cintra, 2010). Há associação entre beleza e empregos de maior prestígio; além disso, as pessoas costumam julgar melhor quem é belo, considerando-os mais inteligentes, com maior competência social e outras qualidades desejáveis (Myers, 2000). 
Atualmente adolescentes modelos são divididos em categorias de atuação na profissão conhecidas como modelos fotográficas e de passarela (fashion). O primeiro grupo caracteriza-se por fazer trabalhos publicitários como fotos para revistas, encartes de moda e, por serem consideradas baixas, não fazem desfiles. Já as modelos fashion realizam desfiles e apresentam coleções de estilistas nas passarelas. Devem ser mais magras e mais altas em relação às modelos fotográficas e podem, também, fazer trabalhos de publicidade (Rodrigues, 2007).

As agências podem captar as adolescentes por meio de um scouter (caçador de talentos), um agente, um booker (profissional que negocia os trabalhos das modelos), um produtor de elenco ou qualquer outra pessoa que esteja envolvida profissionalmente com a contratação de modelos (Libardi, 2004). Muitas vezes vão procurá-las em cidades pequenas, afastadas do eixo Rio-São Paulo.

As adolescentes podem batalhar pelos seus próprios interesses por meio da contratação de uma consultoria, procurando por uma grande agência, fazendo um curso profissionalizante, participando de concursos, workshops, palestras ou procurando agências caça-talentos (Libardi, 2004). Neste sentido, o trabalho juvenil pode contribuir para o desenvolvimento psicossocial, numa perspectiva mais ampla, quando esta experiência laboral consegue fornecer alternativas para a vida da adolescente onde outras estruturas falharam (Liborio \& Ungar, 2010). É possível um desenvolvimento positivo, mesmo estando em um ambiente que exponha o adolescente a múltiplos riscos (Seccombe, 2002). As adolescentes modelos que apresentam este comportamento, denominado por Seccombe (2002) de resiliente, têm a oportunidade de viver experiências múltiplas dentro de um continuum infância-adultícia ou aprendizagem-trabalho (Liborio \& Unger, 2010).

A adolescente estreia oficialmente como modelo quando consegue ter seu "book" (álbum de fotos) disponível numa agência (Guerra, 1997). Muitas modelos iniciam sua profissão precocemente, por volta dos 13 anos. Estas adolescentes deixam a convivência familiar e iniciam um convívio com um grupo profissional; passam a serem responsáveis pelos próprios cuidados e a administrar questões relacionadas à profissão, como a imagem corporal, a alimentação e as finanças (Libardi, 2004; Rodrigues, 2007). Precisam desenvolver recursos estratégicos para tomada de decisões, especialmente aquelas que envolvem seu futuro (Schoen-Ferreira, Aznar-Farias \& Silvares, 2009).

Muitas das que optaram por esta profissão saem de suas cidades ou estados e vêm para os centros onde estão localizadas as principais agências. Deixam de morar com suas famílias, ter convívio com amigos de longa data, frequentar a escola e fazer atividade física e recreativa comuns na adolescência. Quando vêm morar em uma cidade grande, podem precisar ter de dividir seu espaço (casa, quarto) com outras meninas desconhecidas e que são suas concorrentes profissionais (Rodrigues, 2007). As agências alugam casas para as meninas morarem. As modelos ficam com um espaço restrito, tendo de guardar seus objetos pessoais em pequenos armários ou dentro da mala.

Ao chegarem, por volta dos 13 anos de idade, as modelos apresentam uma forma corporal magra e com aspecto infantil, apesar da altura ser superior à média das adolescentes de mesma faixa etária (Rodrigues, 2007). Todas essas mudanças psicossociais podem influenciar na qualidade de vida desse grupo, podendo afetar a percepção dessas adolescentes, o ambiente em que vivem e suas relações interpessoais.

O tema qualidade de vida tem despertado interesse de diversos campos do conhecimento humano, em especial o da saúde, porém, ainda não existe um consenso sobre este construto (Ferraz, 1998). Sobre as definições de qualidade de vida, pode-se afirmar que elas são tão numerosas como os métodos para avaliá-la. Na literatura científica, observam-se inúmeras controvérsias teóricas e metodológicas, principalmente em decorrência da característica multidimensional e subjetiva do construto, e também pelos diversos fatores que interferem na sua construção como objeto de avaliação ou de pesquisa. Assim, o conceito de qualidade de vida está inserido em um campo semântico polissêmico, amplo e genérico (Farquhar, 1995).

Neste trabalho, o termo qualidade de vida está definido como a percepção do indivíduo de sua posição na vida no contexto da cultura e sistema de valores nos quais ele vive e em relação aos seus objetivos, expectativas, padrões e preocupações (Fleck, 2000). É importante que as pessoas se sintam bem psicologicamente, possuam boas condições físicas e sintam-se socialmente integradas e funcionalmente competentes (Fleck e et al., 1999). A promoção da qualidade de vida de adolescentes representa um desafio, pois este segmento da população é considerado bastante vulnerável para enfrentar sozinho as exigências do ambiente (Costa \& Bigras, 2007). O nível de qualidade de vida pode ser estimado por meio do questionário World Health Organization Quality of Life - versão breve WHOQOL-BREF-, proposto pela Organização Mundial de Saúde - OMS em 1995, cuja versão em português foi validada por Fleck et al. (1999)

Poucos estudos foram encontrados associando adolescentes e qualidade de vida utilizando o questionário WHOQOL-BREF (Chen, Wu, \& Yao, 2006). Um desses estudos mostra a comparação da qualidade de vida, saúde mental, estado nutricional de adolescentes em Dhaka, Bangladesh, que viviam em favelas ou não. Por meio do WHOQOL-BREF pode-se perceber pior qualidade de vida devido às condições ambientais (Izutsu, Tsutsumi, Islam, Kato, Wakai, \& Kurita, 2006). O estudo de Chen e Yao (2010) observou que o processo de construção da identidade pessoal estava associado a uma boa qualidade de vida; práticas educativas parentais à qualidade de vida dos jovens (Zimmermann, Eisenmann \& Fleck, 2008).

Adolescentes vivendo longe de sua cultura original podem ter menores escores nos testes de qualidade de vida, como no estudo de Bayram, Thorburn, Dmirhan e Bilgel (2007), no qual observaram escores de qualidade de vida moderados em adolescentes filhos de imigrantes turcos morando na Suécia, mesmo tendo nascido neste país. A qualidade de vida das meninas parece ter sido mais afetada, pois seus escores foram menores que os dos meninos.

As adolescentes modelos passam por mudanças corporais e psicossociais, devem apresentar um repertório de comportamentos exigidos pela profissão, os quais, muitas vezes, podem ser contrários aos valores de suas famílias 
ou às necessidades de seu próprio desenvolvimento. Esses comportamentos podem intervir na qualidade de vida, pois abarcam o ambiente em que a modelo vive e interferem no cumprimento das tarefas da adolescência, como estudar.

Neste sentido, o objetivo deste estudo foi analisar a qualidade de vida de adolescentes modelos por meio do WHOQOL-BREF, comparando com adolescentes não modelos.

\section{Método}

\section{Participantes}

Participaram do estudo adolescentes do gênero feminino com idades de 15 a 19,9 anos, que não estavam grávidas ou que não fossem mães, totalizando 37 modelos oriundas das diversas regiões brasileiras, contratadas por agências da cidade de São Paulo e 37 adolescentes não modelos, estudantes de escolas particulares ou públicas da cidade de São Paulo.

\section{Instrumentos}

Foram utilizados, além de um questionário sóciodemográfico, dois instrumentos: WHOQOL-BREF, para avaliação da qualidade de vida; o Critério Brasil 2008, para avaliação do nível socioeconômico.

O nível de Qualidade de Vida foi estimado por meio do questionário WHOQOL-BREF, proposto pela Organização Mundial da Saúde (WHO, 1995) em 1995, cuja versão em português foi validada por Fleck et al. (1999). Este instrumento é composto de 26 questões e analisa os domínios físico, psicológico, social e ambiental separadamente, propondo um resultado total (qualidade de vida global). A cada domínio, de acordo com as respostas de quem preencheu o questionário, são atribuídos valores que variam de 50 a 100 . Quanto maior a pontuação, melhor a qualidade de vida. $\mathrm{O}$ domínio físico refere-se às possíveis dores e desconfortos, fadiga, sono e repouso; no domínio psicológico avalia-se a autoestima, imagem corporal e aparência, sentimentos positivos e negativos, assim como capacidade para aprender e se concentrar; o domínio social refere-se ao nível de independência, considerando-se a mobilidade, capacidade para o trabalho, bem como dependência de medicação ou de tratamentos do indivíduo; e, no domínio ambiental são analisadas as relações pessoais, atividade sexual, recursos financeiros, segurança física e proteção, transportes, oportunidades de lazer, religião etc.

As questões do WHOQOL-BREF foram formuladas para uma escala de respostas do tipo Likert, de cinco pontos, com escalas para intensidade (nada - extremamente), capacidade (nada - completamente), frequência (nunca - sempre) e avaliação (muito insatisfeito - muito satisfeito; muito ruim - muito bom).

A avaliação socieconômica foi realizada segundo os critérios estabelecidos pela Associação Brasileira de Empresas de Pesquisa - ABEP (2008). O Critério de Classificação Econômica Brasil - Critério Brasil 2008 - tem a função de estimar o poder de compra das pessoas e famílias urbanas, e classifica a população em oito classes, a saber: A1, A2, B1, $\mathrm{B} 2, \mathrm{C} 1, \mathrm{C} 2$, D e E; sendo as classes A com maior poder de compra e a $\mathrm{E}$ com menor.

Um questionário sociodemográfico, elaborado especialmente para este estudo, foi utilizado para caracterização da população de modelos adolescentes a ser estudada (data de nascimento, profissão, tempo de profissão, idade com que iniciou a profissão, data da última menstruação - mês e ano, estatura, massa corporal, idade da menarca - mês e ano). Para adolescentes não modelos o mesmo questionário foi aplicado excluindo as questões referentes à profissão.

\section{Procedimentos}

Após aprovação do estudo pelo Comitê de Ética em Pesquisa da Unifesp, solicitou-se autorização das agências de modelo para proceder ao estudo. As modelos foram visitadas em suas moradias, para explicação do trabalho e distribuição do Termo de Consentimento Livre e Esclarecido. Aquelas que aceitaram participar do estudo responderam aos questionários em horário previamente acordado, em suas residências.

Também foi solicitada a autorização de duas escolas para proceder ao estudo, com consentimento dos pais. As adolescentes, do nono ano do Ensino Fundamental ao $3^{\circ}$ ano do Ensino Médio, responderam aos instrumentos em horário determinado pela escola, na presença da pesquisadora e de um professor. $\mathrm{O}$ pareamento dos grupos foi realizado pelo Critério Brasil 2008 e idade, compondo 37 pares modelo-não modelo. Para a realização do pareamento pelo nível socioeconômico, foram avaliadas 66 modelos e 46 não modelos, conforme descrito na Tabela 1.

Tabela 1. Distribuição da Amostra Original de Acordo com o Resultado do Critério Brasil 2008.

\begin{tabular}{|c|c|c|c|c|}
\hline Classe segundo o & Modelo & Não-modelo & Total & Pareamento \\
\hline Critério Brasil* & N (\%) & $\mathrm{N} \quad(\%)$ & N $\quad(\%)$ & $\mathrm{N} \quad(\%)$ \\
\hline $\mathrm{A} 2$ & $6(9,09 \%)$ & $5(10,86 \%)$ & $11(9,82 \%)$ & $5(13,5 \%)$ \\
\hline B1 & $9(13,63 \%)$ & $16(34,78 \%)$ & $25(22,32 \%)$ & $9(24,3 \%)$ \\
\hline B2 & $17(25,75 \%)$ & $19(41,30 \%)$ & $36(32,14 \%)$ & $17(45,9 \%)$ \\
\hline $\mathrm{C} 1$ & $29(43,93 \%)$ & $4(8,69 \%)$ & $31(27,67 \%)$ & $4(10,8 \%)$ \\
\hline $\mathrm{C} 2$ & $5(7,57 \%)$ & $2(4,34 \%)$ & $9(8,03 \%)$ & $2(5,4 \%)$ \\
\hline Total & $66(100 \%)$ & $46(100 \%)$ & $112(100 \%)$ & $37(100 \%)$ \\
\hline
\end{tabular}

*Classe A2 apresenta maior poder de compra em relação às seguintes. Classe $\mathrm{C} 2$ com menor poder em relação às anteriores. 
A maior parte das modelos encontrava-se no critério $\mathrm{C}$ (1 ou 2) e das não modelos no critério B (1 ou 2). Puderam ser formados 37 pares.

\section{Análise de Dados}

O pressuposto de distribuição normal dos dados foi verificado pelo teste de Kolmogorov-Smirnov. Foi utilizada estatística descritiva por meio de análise de frequência, média e desvio-padrão. Para as comparações foi utilizada estatística paramétrica, por meio do teste $\mathrm{t}$ de Student, pois todas as variáveis apresentaram distribuição normal.

O teste de Kolmogorov-Smirnov mostrou que em todos os domínios a distribuição era normal, o que permitiu utilizar estatística paramétrica, por meio de média e desvio-padrão, e comparar os grupos utilizando-se o teste $\mathrm{t}$ de Student para amostras independentes.

\section{Resultados}

De acordo com os resultados, as participantes do estudo encontravam-se com uma boa qualidade de vida (ver Tabela 2 ), embora algumas adolescentes apresentassem escores Tabela 2. Comparação entre Modelos e Não-modelos, por Resultado Médio Obtido nos Domínios do WHOQOL-BREF.

\begin{tabular}{lcc}
\hline Domínio & $\begin{array}{c}\text { Modelo } \\
\text { Média } \pm D P\end{array}$ & $\begin{array}{c}\text { Não modelo } \\
\text { Média } \pm D P\end{array}$ \\
\hline Físico & $73,165 \pm 13,95$ & $68,655 \pm 10,67$ \\
Psicológico & $72,973 \pm 12,64$ & $66,897 \pm 11,61^{*}$ \\
Social & $71,165 \pm 13,26$ & $73,419 \pm 13,29$ \\
Ambiental & $61,751 \pm 11,15$ & $61,243 \pm 12,09$ \\
Global & $69,697 \pm 9,92$ & $66,486 \pm 8,15$ \\
\hline
\end{tabular}

*valor significativo $\mathrm{p}<0,05$

médios bastante baixos. Em geral, o grupo de modelos obteve médias superiores ao grupo de não modelos, sendo esta diferença significativa apenas no domínio psicológico.

\section{Discussão}

Como observado na Tabela 1 , às modelos deste estudo apresentam um poder de compras característico da classe $\mathrm{C}$, que não é considerado classe média, porém está em ascensão. Embora a família ainda não possa manter os estudos dos filhos, há o sonho de uma melhora no estilo de vida. Como outras profissões glamorosas que não exigem um investimento familiar muito grande, a profissão de modelo surge como uma possibilidade de ascensão social e econômica (Rodrigues, 2007).
Ao se parear o grupo de estudantes com o grupo de modelos profissionais, houve a preocupação de se entrevistar adolescentes nas mesmas escolas e séries que as modelos frequentavam. Entretanto, houve uma perda de 43,93\% no grupo estudo, por não se encontrar um controle cuja família apresentasse o mesmo poder de compra. Provavelmente, isto se deve ao fato das modelos serem oriundas de regiões rurais em que o nível de consumo costuma ser menor que daqueles de regiões urbanas, assim como o nível educacional do chefe da família (ABEP, 2008). Rodrigues $(2003,2007)$ discute a dificuldade de se encontrar controles para estas adolescentes, dado as peculiaridades físicas que a profissão exige. Observamos que, em relação às características socioeconômicas, elas também são bastante diferentes de seus pares, o que nos leva a concordar com Schoen-Ferreira, Aznar-Farias e Silvares, (2010), quando afirmam que a adolescência é um fenômeno universal, mas com particularidades de acordo com o contexto.

Embora precisem lidar com a exposição maciça de seu corpo pela mídia e com a possibilidade de sentirem-se usadas como um objeto, sua presença em um espaço público alimenta o sonho que as fez saírem de suas casas e buscarem uma agência de modelos (Rodrigues, 2003). As modelos que participaram deste estudo estão vivendo longe de suas cidades de origem, em uma cultura bem diversa da que foram educadas. Entretanto, com observado na Tabela 2, diferente do estudo de Bayram, Thorburn, Dmirhan e Bilgel (2007), elas apresentaram uma boa qualidade de vida. Este resultado talvez se deva, como colocado por Chen e Yao (2010), ao processo de construção de identidade. A identidade funciona com um sistema de autorregulação, processando as informações, gerenciando as impressões e selecionando os comportamentos apropriados (Schoen-Ferreira, Aznar-Farias \& Silvares, 2009).

As famílias das modelos não podem ser acusadas, como diriam Costa e Bigras (2007), de não cumprirem sua função protetora, colocando suas filhas em situações de risco (Schoen-Ferreira \& Vitalle, no prelo). Ao contrário, parece que essas famílias abriram espaço para que as adolescentes busquem seus sonhos e se tornem adultos maduros e independentes. Embora estejam longe dos pais, trazem consigo o sentimento de amor e proteção o qual, provavelmente as ajude a enfrentar as vicissitudes de sua escolha (Zimmermann, Eisenmann \& Fleck, 2008).

Em relação ao domínio Psicológico, as adolescentes modelos tiveram uma média significantemente mais alta que as não modelos. Cabendo ressaltar que as perguntas relacionadas a este domínio referem-se a autoestima, imagem corporal e aparência.

O indivíduo desenvolve sua autoestima a partir do modo como percebe a si mesmo e esta percepção é alimentada pelas experiências cotidianas. Em um mundo que valoriza a aparência (Calatayud Arenes \& Serra Desfilis, 2009, Sarmento, Schoen-Ferreira, Medeiros \& Cintra 2010), as modelos já apresentam uma grande vantagem. Provavelmente elas são invejadas pelas suas colegas de escola. Inúmeros estudos vêm demonstrando que a aparência é importante no relacionamento social, especialmente para o sexo feminino (Myers, 2000). A beleza, no imaginário popular, está relacionada a 
outros aspectos positivos do comportamento humano, como a bondade e a inteligência (Myers, 2000, Aronson, Wilson \& Akert, 2002). Sabemos que a atração física não supera outras qualidades, mas é possível que as adolescentes modelos tenham mais facilidade em algumas áreas de sua vida, por serem belas.

Apesar de terem deixado suas famílias de origem e estarem morando bastante longe, o que poderia levar a maiores dificuldades na vida diária por não contarem com a proteção de um adulto com vínculo afetivo (Costa \& Bigras, 2007), e, portanto, índices mais baixos na avaliação da qualidade de vida, isto não ocorreu neste estudo. Provavelmente a maturidade desenvolvida por estas meninas decorrente da necessidade de viverem sem um adulto protetor junto com a realização de um sonho e a certeza de que precisam trabalhar para alcançar seus objetivos (Schoen-Ferreira, Aznar-Farias \& Silvares, 2009), as impulsiona para comportamentos mais adultos e responsáveis, o que reflete numa boa qualidade de vida. Cabe ressaltar aqui que essas meninas são em grande parte provenientes de famílias de baixa renda e com pouco acesso a eletrodomésticos que facilitam a vida no lar ou a empregada mensalista (ABEP, 2008). Muito provavelmente, foram educadas desde pequenas a dividirem com seus pais as tarefas tanto domésticas quanto as que garantem o sustento da família.

Quase tudo na vida de uma modelo que deixou sua cidade e veio morar só em uma metrópole levaria a pensarmos na instalação de comportamentos psicopatológicos, como o isolamento ou a depressão. Entretanto, parece que a presença de objetivos claros serve como fator protetor para o desenvolvimento de maior repertório comportamental adequado à construção de seu plano de vida. Neste sentido, concordamos com Liborio e Ungar (2010), em que o trabalho juvenil precisa ser estudado de uma perspectiva maior. As adolescentes modelos profissionais que conseguem aproveitar as vicissitudes a que estão expostas e transformarem-nas em oportunidades para crescimento, ou seja, utilizando estratégias e recursos de forma resiliente (Seccombe, 2002), parecem apresentar, então, uma melhor qualidade de vida.

O único domínio em que o grupo de modelos obteve escores médios mais baixos que o grupo controle foi o domínio social, que envolve independência e capacidade para o trabalho. Entretanto, esta diferenciação não foi estatisticamente significante. Acreditamos que, neste caso específico, como o grupo controle era composto por adolescentes que não trabalhavam, as questões do WHOQOL-Bref não abrangiam a experiência destes indivíduos, enquanto que o trabalho é uma preocupação constante na vida da adolescente modelo. Assim como a falta de ocupação profissional. O desemprego - aqui entendido como não ter sido selecionada para um trabalho - ou a convivência na mesma moradia com outras concorrentes podem ter um peso maior nas respostas deste grupo ao instrumento. $\mathrm{O}$ emprego de modelo não é fixo, não consiste em um único contrato, mas envolve constantes seleções para pequenos trabalhos no decorrer do ano (Rodrigues, 2007). Desta forma, a adolescente modelo vive constantemente o estresse buscar um trabalho, que sabe que será por um curto período, e nem sempre remunerado.
Impulsionadas pelas estratégias de sobrevivência pessoal, as jovens se sentem compelidas a precocemente deixarem suas famílias e buscarem uma ocupação inconstante e que não gera segurança. Vivem no dilema do "agora ou nunca", ou seja, a carreira de modelo é bastante curta, necessitando que a menina faça sucesso ainda na adolescência ou não terá futuro. Ao mesmo tempo, esta carreira dificulta o acesso das jovens a outra profissão - estudando ou tendo algum trabalho paralelo -, pois exige disponibilidade integral. É neste momento que o trabalho juvenil pode ser um entrave para o desenvolvimento integral do indivíduo (Liborio \& Ungar, 2010), inclusive limitando seu futuro profissional.

A busca por ser selecionada para um trabalho é imperiosa, fazendo com que os percalços do caminho sejam encarados como entraves necessários para se alcançar o objetivo (Schoen-Ferreira, Aznar-Farias \& Silvares, 2009). Não é necessário lutar contra eles, mas aceitá-los da forma mais tranquila que conseguirem. A modelo é uma presença feminina na mídia, e esta adolescente luta para conquistar o seu espaço e ser reconhecida dentro da profissão que resolveu abraçar.

Em geral, as adolescentes modelos participantes deste estudo apresentavam uma qualidade de vida semelhante a qualquer outra adolescente. Questões sobre o lazer, utilização de transporte público, vida sexual ou religião são vivenciadas conforme sua inserção histórica e cultural, ou seja, existem variadas formas de viver a adolescência, de acordo com o gênero, o grupo social ou localização geográfica (Pfeifer, Martins \& Santos, 2010; Schoen-Ferreira, Aznar-Farias \& Silvares, 2010).

Este trabalho esteve interessado em observar semelhanças e diferenças entre modelos profissionais e não modelos, enfocando a qualidade de vida, a fim de que o conhecimento advindo deste estudo possa promover um melhor entendimento desta fase da vida e sirva de linha de base para futuros estudos com objetivos semelhantes, estudando diferentes formas de viver a adolescência, ou seja, com a participação de grupos em diferentes situações.

\section{Referências}

Aronson, E., Wilson, T. D., \& Akert, R. M. (2002). Psicologia social. Rio de Janeiro: LTC.

Associação Brasileira de Empresas de Pesquisa - ABEP. (2008). Critério padrão da classificação econômica Brasil/2008. Retrieved from http://www.abep.org/ codigosguias/ Criterio_ Brasil_2008.pdf .

Bayram, N., Thorburn, D., Demirhan, H., \& Bilgel, N. (2007). Quality of life among Turkish immigrants in Sweden. Quality of Life Research, 16(8), 1319-1333.

Calatayud Arenes, M. P., \& Serra Desfilis, E. (2009). Las relaciones de amor em los adolescentes de hoy. Barcelona: Octaedro.

Chen, K. H., \& Yao, G. (2010). Investigating adolescent healthrelated quality of life: from a self-identity perspective. Social Indicators Research, 96(3), 403-415.

Chen, K. H., Wu, C. H., \& Yao, G. (2006). Applicability of the WHOQOL-BREF on early adolescence. Social Indicators Research, 79, 215-234. 
Costa, M. C. O., \& Bigras, M. (2007). Mecanismos pessoais e coletivos de proteção e promoção da qualidade de vida para a infância e adolescência. Ciência \& Saúde Coletiva, 12(5), 1101-1109.

Farquhar, M. (1995). Definitions of quality of life: A taxonomy. Journal of Advanced Nursing, 22, 502-508.

Ferraz, M. B. (1998). Qualidade de vida: conceito e um breve histórico. Jovem Médico, 3(4), 219-222.

Fleck, M. P. A, Leal, O. F., Lousada, S., Xavier, M., Chachamovich, E., Vieira, G., Santos, L., \& Pinson, V. (1999). Desenvolvimento da versão em português do instrumento de avaliação de qualidade de vida da OMS (WHOQOL-100). Revista Brasileira de Psiquiatria, 21, 19-28.

Fleck, M. P. A. (2000). O instrumento de avaliação de qualidade de vida da Organização Mundial da Saúde (WHOQOL-100): características e perspectivas. Ciência \& Saúde Coletiva, 5, 33-38.

Guerra, L. (1997). Retrato de Modelo. Porto Alegre: Lp\&m-Zero Hora.

Izutsu, T., Tsutsumi, A., Islam, A., Kato, S., Wakai, S., \& Kurita, H. (2006). Mental health, quality of life, and nutritional status of adolescents in Dhaka, Bangladesh: Comparison between an urban slum and no-slum area. Social Science \& Medicine, 63(6), 1477-1488.

Libardi, M. (2004). Em busca da fama: profissão modelo. São Paulo: Senac.

Liborio, R. M. C., \& Ungar, M. (2010). Children's labour as a risky pathway to resilience: Children's growth in contexts of poor resources. Psicologia: Reflexão e Crítica. 23(2), 232-242.

Mota, C. P., \& Matos, P. M. (2009). Apego, conflito e auto-estima em adolescentes de famílias intactas e divorciadas. Psicologia: Reflexão e Crítica, 22(3), 344-352.

Myers, D. G. (2000). Psicologia social. Rio de Janeiro: LTC.

Noronha, A. P. P., \& Ambiel, R. A. M. (2006). Orientação profissional e vocacional: análise da produção científica. PsicoUSF, 11, 75-84.

Pfeifer, L. I., Martins, Y. D., \& Santos, J. L. F. (2010). A influência socioeconômica e de gênero no lazer de adolescentes. Psicologia: Teoria e Pesquisa, 26(3), 427-432.

Rodrigues, A. M. (2003). Perfil nutricional de modelos adolescentes brasileiras. Dissertação de Mestrado, Universidade Federal de São Paulo, São Paulo.
Rodrigues, A. M. (2007). Análise comparativa da composição corporal, densidade mineral óssea, taxa metabólica em repouso e ingestão alimentar de adolescentes modelos e não modelos. Tese de Doutorado, Universidade Federal de São Paulo, São Paulo.

Sarmento, A. S. L., Schoen-Ferreira, T. H., Medeiros, E. H., \& Cintra, I. P. (2010). Avaliação dos sintomas emocionais e comportamentais em adolescentes obesos. Estudos e Pesquisas em Psicologia, 10(3), 833-847.

Schoen-Ferreira, T. H., \& Vitalle, M. S. S. (no prelo). Contextos sociais do adolescente: família, amigos, escola e trabalho. In M. B. Moraes (Ed.), Diagnóstico e tratamento em pediatria (pp. 115-118). Barueri, SP: Manole.

Schoen-Ferreira, T. H., Aznar-Farias, M., \& Silvares, E. F. F. (2010). Adolescência através dos séculos. Psicologia: Teoria e Pesquisa, 26(2), 227-234.

Schoen-Ferreira, T. H., Aznar-Farias, M., \& Silvares, E. F. F. (2009). Desenvolvimento da identidade em adolescentes estudantes do ensino médio. Psicologia: Reflexão e Crítica, 22(3), 326-333.

Seccombe, K. (2002). "Beating the odds" versus "Changing the odds": Poverty, resilience, and family policy. Journal of Marriage and Family, 64(2), 384-394.

World Health Organization (WHO). (1995). Adolescents. Physical status: The use and interpretation of anthropometry (Technical Report Series 854). Genebra; WHO.

Zimmermann, J. J., Eisenmann, M. R., \& Fleck, M. P. (2008). Is parental rearing an associated factor of quality of life in adulthood?. Quality of Life Research, 17(2), 249-255. 